\title{
THEORETICAL ASPECTS OF THE PREDICTIONAL INSTRUMENTATION FOR APPLICATION IN THE STATE REGULATION OF THE PARTICIPANTS RELATIONSHIPS IN THE ELECTRICITY MARKET
}

\author{
Anastasiia KOLIESNICHENKO ${ }^{1}$ \\ National Technical University "Kharkiv Polytechnic Institute”, Ukraine
}

\begin{abstract}
The article presents the problems of current trends in the development of the electric power industry in conditions of increasing capital concentration, informing the economy and increasing the dynamics of the movement of significant amounts of cash flows which require a permanent analysis of the current situation and the necessary adjustment and / or modification of the parameters for the regulation of energy markets. From this article it can be concluded that the task of constructing forecasts acquires high relevance in many subject areas. It is an integral component of the daily work of modern social and economical systems. The interrelations of the subjects of the electric energy market are the one of the its most important institutions. The purpose of this article is to study the theoretical basis for the adaptive application of forecasting methods in the functioning of energy markets. In order to avoid the dominance of commercial interests of certain groups of participants in the energy market, the emergence of price distortions in the market that have a destructive effect on obtaining potential benefits from the introduction of competitive mechanisms, the key to finding the most effective and economical way to solve these problems is the use of systemic regulation of emerging deviations in achieving target milestones especially in the context of reform. The informational and analytical support formation for the performance of the functions assigned to the regulatory apparatus requires the use of forecasting methods and approaches to preserve the dynamic development of the electric energy market and to maintain the balance of interests of all its subjects in the conditions of reform. The article uses a number of methods: classification and systematization in the analysis of scientific methods for forecasting and planning social and economic processes, logical generalization when reviewing existing modeling methods and assessing their effectiveness in the energy markets, the use of a constructive approach in the study of factors affecting the resulting index. Finally the obtained theoretical and scientifically applied results of the research make it possible to formulate the necessary theoretical and methodological basis for improving the instrumental basis for modeling the relationships between the subjects of the electric energy market, which can be used in forecasting in the field of regulation of phenomena and processes in the energy markets.
\end{abstract}

Key words: the regulation, energy market, energy market participants, forecasting methods.

JEL Classification: C3, C53, P18, Q4, Q48

\section{1. Введение}

Концепция государственного регулирования основывается на комплексе методических мероприятий, направленных на построение эффективного механизма обеспечения внутренних и внешних функций государства по всем направлениям национального хозяйства. Ключевыми инструментами, позвомяющими формировать ресурсно-управленческую систему и сохранять баланс интересов субъектов на рынке, которые характеризуются разной структурой капитала и неодинаковой чувствительностью к регумяторному влиянию в контексте неравномерного распределения прав собственности, выступают пиановые и прогнозные технологии и процедуры.

Выбор соответствующей стратегии и реализация процесса принятия конкретных конструктивных решений органами законодательной власти, органами местного самоуправмения по регулированию социально-экономических процессов Аолжны не только опираться на результаты ретроспективного анализа, но и в значительной степени учитывать прогнозы и их корректировки, составленные с целью достижения целевых ориентиров развития, модернизации или реформирования. Это обусловливает необходимость

Corresponding author:

${ }^{1}$ Department of Accounting and Analysis, National Technical University „Kharkiv Polytechnic Institute”.

E-mail: anastasihpi@gmail.com 
обеспечения качественного и квамифицированного подхода к прогнозированию текущей экономической ситуации и перспективных показателей в различных отраслях народного хозяйства.

ОАними из перспективных путей решения проблемы электроэнергетической зависимости страны остаются развитие альтернативной энергетики и наращивание экспорта электрической энергии.

\section{2. Обзор текущей ситуации на рынке электроэнергии Украины}

Общий объем технически возможного энергетического потенциама возобновмяемых источников энергии (Аалее - ВИЭ) в Украине ветра, солнца, воды, биомассы - в современных условиях эквивалентный 81 мин т условного топиива, что составляет около $40 \%$ годового спроса на электроэнергию (Gonta, 2015).

ОАнако, несмотря на общедоступный характер ВИЭ, бкагоприятные природно-кмиматические преАпосылки Аля активизации их использования и имеющуюся тенденцию количественного роста в совокупном товарном отпуске электроэнергии в оптовый рынок, их Аоля в структуре генерации электроэнергии в нашей стране остается несущественной, в частности, в 2016 г. суммарный объем электроэнергии, произведенной из альтернативных источников, составим 1,3\% всего производства (Otchet, attachmen 2.3.8, 2016).

После некоторого роста экспорта эмектроэнергии в 2012-2013 гг. одновременно с объемами общего производства, в 2014 г. Аанный показатемь снизился на $13 \%$ по сравнению с 2013 г. и в 2015 г. Украина сократила экспорт электроэнергии на 54,8\%, что

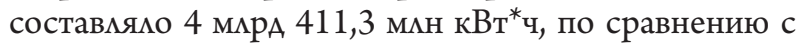
2014 г. Ао 3 ммрА 641,6 млн кВт ${ }^{*} ч$ (Otchet, attachmen 2.2.4, 2016). Такая ситуация сложилась главным образом из-за прекращения поставок электрической энергии в Молдову и Республики Беларусь с конца осени 2014 г., что было обусловлено острым Аефицитом энергетического угля. Кроме того, с целью сбалансирования работы ОЭС Украины в условиях Аефицита топлива и гидроресурсов по состоянию на начало 2015 г. был ограничен экспортный потенциац «Бурштынского острова», через который осуществляется экспорт электроэнергии в страны ЕС (Ekonomika. Energetika, 2015).

В 2016 г. Украина увеличила экспорт электроэнергии на $11 \%$ по сравнению с 2015 г. На протяжении 2015-2016 гг. экспорт осуществиялся в страны ENTSO-E.

Оценивая неАостатки и преимущества внеАрения конкретных направлений совершенствования системы регулирования современных энергорынков, целесообразно взвешивать внутренние условия и соотношение Аомей эмектроэнергии, поступающей в ОЭС от размичных типов электростанций с учетом Аинамики их внутриструктурных трансформаций.

Традиционно Аля украинской ОЭС стратегически формирующей основой остается атомная энергия, которая в количественном выражении по результатам 2016 г. составила более половины всей генерации. При этом необходимо отметить, что важным фактом стало уменьшение Аоли электроэнергии, производимой АЭС, в структуре всей отпущенной эмектроэнергии в ОРЭ при общем уменьшении производства. Так, в 2016 г. всеми электростанциями

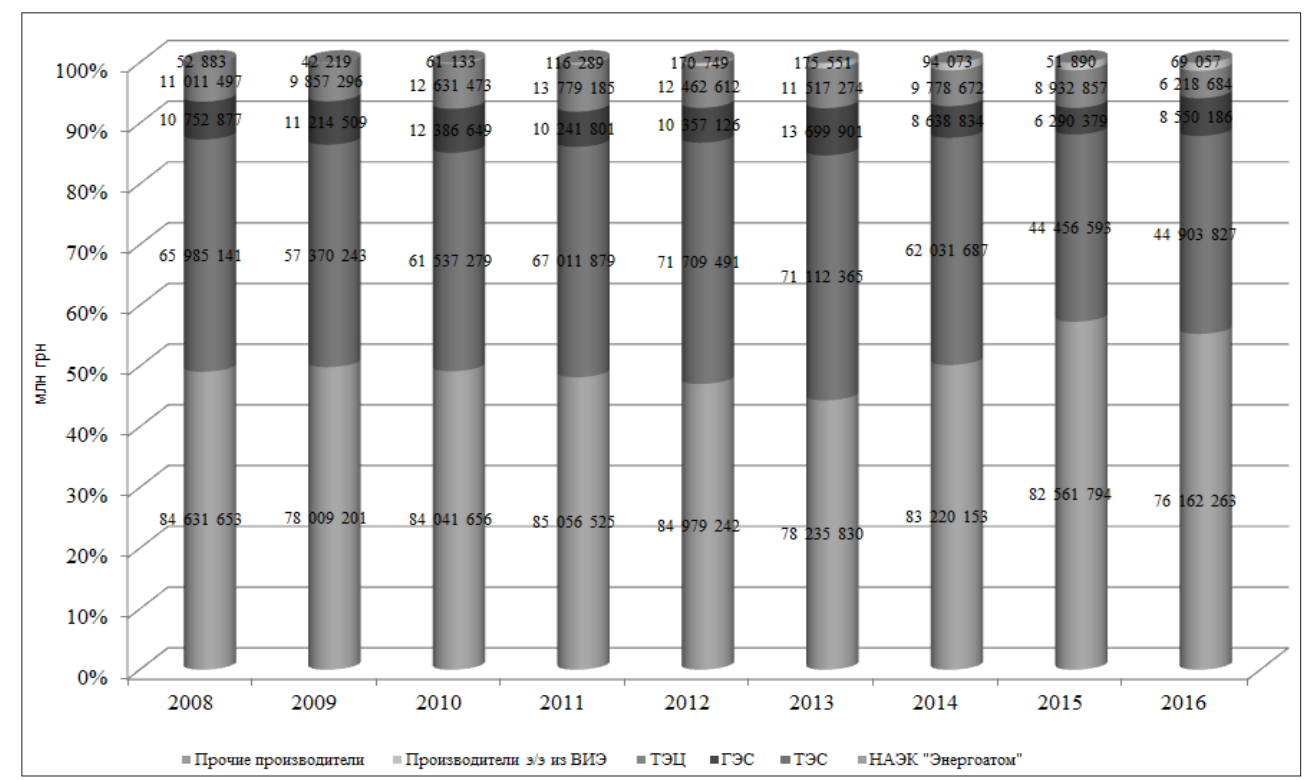

Рис. 1. Аинамика изменения структуры генерации экектроэнергии в Украине

Источник: Otchet o rezultatah deyatelnosti Natsionalnoy komissii, osuschestvlyayuschey gosudarstvennoe regulirovanie $v$ sferah energetiki i kommunalnyih uslug, $v 2016$ godu 
страны было сгенерировано эмектрической энергии на 1,9\% меньше, чем в 2015 г. при сокращении Аоли «атомной» энергии с 56,9\% в 2015 г. Ао 53,9\% в 2016 г. (Otchet, attachmen 2.3.8; 2.3.10, 2016).

\section{3. Актуальные вопросы и релевантные заАачи прогнозирования работы рынков эмектрической энергии}

Эффективность государственного регулирования Аеятельности субъектов рынка электрической энергии зависит от анациза структуры генерации и потребления Аанного товара и Аолжна не только учитывать факторы, которые определяют системные изменения и поведение участников рынка, но и проводить мониторинг их вАияния на внутренние закономерности и взаимодействие с взаимосвязанными институтами в плоскости аналогичных отраслевых проблем на макроуровне. То есть, актуальным остается вопрос иАентификации и применения действенных методов и моделей в реальных условиях функционирования энергорынка, что будет способствовать налаживанию механизма государственного регулирования хозяйственной Аеятельности его субъектов с учетом колебаний и нестабильности внешней среАЫ.

Анализ послеАних исслеАований и публикаций. Проблематика построения стратегических планов и выбора наиболее эффективной комбинации инструментов с целью осуществления перспективного анамиза функционирования мировых энергорынков, выделения отдельных аспектов и определения закономерностей влияния государственных решений на социально-экономические и внутриотраслевые процессы, которые основываются на результатах разработанных прогнозов, широко раскрыта в исследованиях отечественных ученых. Наиболее значительных Аостижений в этой сфере добились в своих работах: Grachova, 2013, Danilov, Leonenkov, 2005, Muratova, 2009, Tihonov, 2006 и ар.

Так, Грачева Е. И. (Grachova, 2013) использует регрессионный анализ при опреАелении и прогнозировании потерь электроэнергии при ее транспортировке с целью повышения точности расчетов. В рамках Аанного подхода автором была построена вероятностно-статистическая модель, которая позволяет учитывать Аинамику изменений эмектрических сетей путем увеличения количества описательных характеристик, обусловливает роль первых при многократном использовании.

Изучению проблем планирования и прогнозирования работы рынков электрической энергии, мощности и вспомогательных услуг с применением различных методов и эконометрических моделей, а также регулирования и координации поведения участников таких рынков посвящено много научных трудов зарубежных авторов, среди которых: Catalão, 2005,
2007, Cuaresma, 2004, C. Fezzi 2007, Kristiansen, 2012, Mazengia, 2008, Murthy, 2014, Szkuta, 1999 и ар.

Необходимо отметить, что моделирование цен в сфере электроэнергетики - одно из наиболее распространенных направлений прогнозирования показателей функционирования мировых энергорынков (Cuaresma, 2004; Draper \& Smith, 1981; Escribano, Pẽna, \& Villaplana, 2002; Jingfei Yang, 2006; Kristiansen, 2012; Nan, 2009; Panagiotelis \& Smith, 2008). В частности, авторы работы (Cuaresma, 2004) подчеркивают, что мучшие результаты и точность прогнозов при моделировании стратегий повременного учета электрической энергии по спотовым ценам обеспечивают минейные одномерные модели временных рядов. Автор в работе (Kristiansen, 2012) раскрывает ацгоритм и Аоказывает эффективность применения регрессионных и авторегрессионных моделей с определением величины средней абсолютной ошибки при анализе и прогнозировании почасовой цены на электроэнергию, которая формируется на рынке Nord Pool. Практическое применение метода искусственных нейронных сетей при построении прогнозов ценового выражения и оценке потребления электроэнергии освещены исслеАователями в работе (Szkuta, 1999).

Учитывая степень раскрытия перечисленных проблем в научно-экономической митературе, необходимо отметить, что комплекс вопросов, связанных с методикой воспроизвеАения регулирующей функции государства в контексте взаимоотношений участников энергорынка, требует Аальнейшего рассмотрения.

Целью статьи выступает систематизация методического инструментария составцения планов и прогнозов экономических процессов, обзор кмючевых вопросов и анализ теоретических аспектов применения метода корремяционно-регрессионного анализа Аля выявления факторов, которые оказывают наибольшее влияние на структуру электроэнергетики, что позволит увеличить степень конкретизации и обоснованности Аальнейших исследований в области методики и рычагов государственного регулирования взаимоотношений субъектов электроэнергетического рынка.

\section{4. Теоретические основы модемирования взаимоотношений субъектов энергорынка как фунаамент построения практического прогностического инструментария в области госуАарственного регумирования}

ПоА госуАарственным прогнозированием социально-экономического развития понимают научно обоснованное преАвидение приоритетных направлений и векторов развития страны, отАельных отраслей экономики или аАминистративно-территориальных еАиниц, возможного состояния экономики и соци- 
альной сферы в перспективе, а также определение амьтернативных путей и сроков достижения обозначенных параметров социально-экономического развития (Zin, 2007).

Экспериментальные исследования процессов на рынке электрической энергии получили широкое распространение на различных этапах становления взаимоотношений его субъектов: от разработки стратегий обновления отАельных секторов электроэнергетики до проектирования и фактической экспиуатации электроэнергетической инфраструктуры. Математическая обработка массивов текущей информации обусловмивает необходимость подбора эмпирических формул, которые позволят установить связь Аинамики одного параметра с Аругим.

В настоящее время насчитывается более ста классов моделей, предназначенных Аля прогнозирования. Количество общих классов моделей, которые повторяются в разных вариациях, меньше. ОАна часть модемей и и соответствующие им методы образуют комплекс отАельных процеАур прогнозирования; Аругая часть преАставмяет собой набор опреАеленных приемов, которые отличаются от базовых или Аруг от Аруга количеством частных способов и последовательностью их применения (Tihonov, 2006).

Процессы Аерегумяции, которые происходят в последние годы на значительной части рынков экектрической энергии по всему миру, обусловливают возрастание роли моделирующих инструментов при составлении операционных и стратегических прогнозов их функционирования. Аеятельность участников энергорынков изучается с помощью многочисленных моделей, которые охватывают как минейные временные ряды и многофакторные регрессионные модели, так и компиексные нелинейные модели. ОАнако, опыт построения прогнозов свидетельствует о применении на практике смешанных Подходов к моделированию (Nan, 2009).

Критериями обеспечения точности прогнозов могут выступать различные факторы, к ключевым из них относятся: специфика модели, алгоритм реализации и прогнозный периоА (Nan, 2009).
Наиболее часто в научной митературе, посвященной анализу процессов, происходящих на электроэнергетических рынках, по временному признаку прогнозирование делят на Аолго-, среАне- и краткосрочное. Так, при составлении прогнозов в ценовой плоскости формирования взаимоотношений межАу участниками рынка выдемяют три вида прогнозирования, которые представлены на рис. 2 (Murthy, 2014).

На основе анацитического обзора научных работ авторов в области моделирования (Grachova, 2013, Danilov, Leonenkov, 2005, Muratova, (2005), Zin, 2007) были систематизированы методы прогнозирования и планирования, широко применяемые в исслеАовании социально-экономических явлений и процессов. Их классификация преАставлена на рис. 3 .

Формализованные, или математические методы прогнозирования основаны на получении количественных результатов вычислений, используемых при решении хорошо структурированных и частично слабоструктурированных проблем Аля оценки вариантов решений, выбора и обоснования наиболее оптимального из них. Неформализованные методы, или эвристические применяются при решении сложных слабоструктурированных и неструктурированных проблем Аля генерирования вариантов решений, их анализа и оценки с целью выбора и обоснования наилучшего.

На практике раскрытие многих вопросов, стоящих перед исследователями в размичных отраслях экономики, требует установки и анализа соотношения межАу Авумя и большим количеством переменных. Амя этих целей часто используют регрессионный анализ (Draper, 1981).

Регрессия получает широкое распространение при решении задач прогнозирования и управления. Целью регрессионного анализа выступает опредемение зависимости межАу исходной переменной и множеством внешних факторов (регрессоров). При этом коэффициенты регрессии могут быть опреАелены по методу наименьших квадратов (Аалее МНК) или методом максимального правдоподобия (Draper, 1981).

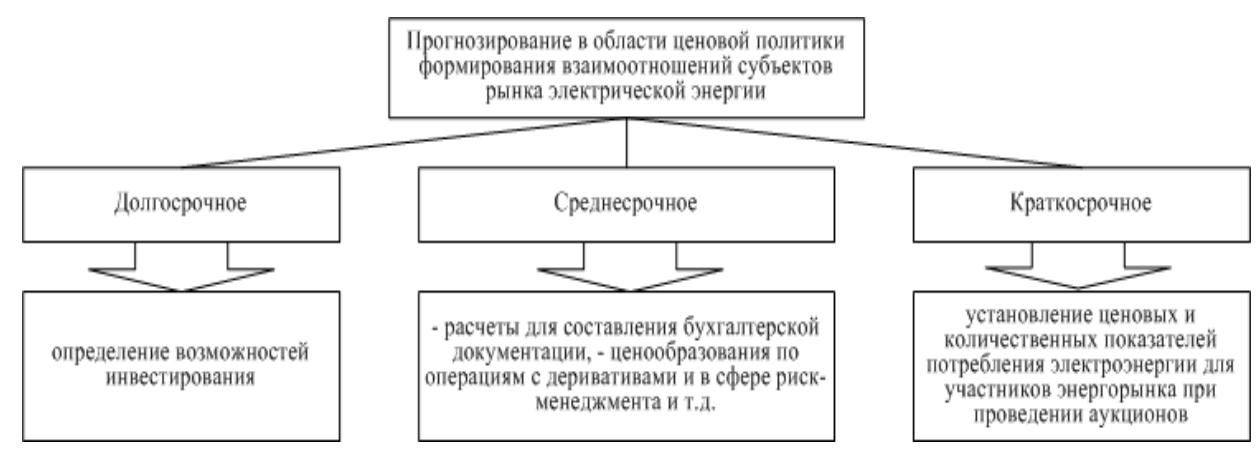

Рис. 2. Характер прогнозирования в области ценовой политики формирования взаимоотношений субъектов рынка эмектрической энергии 
Vol. 3, No. 2, 2017

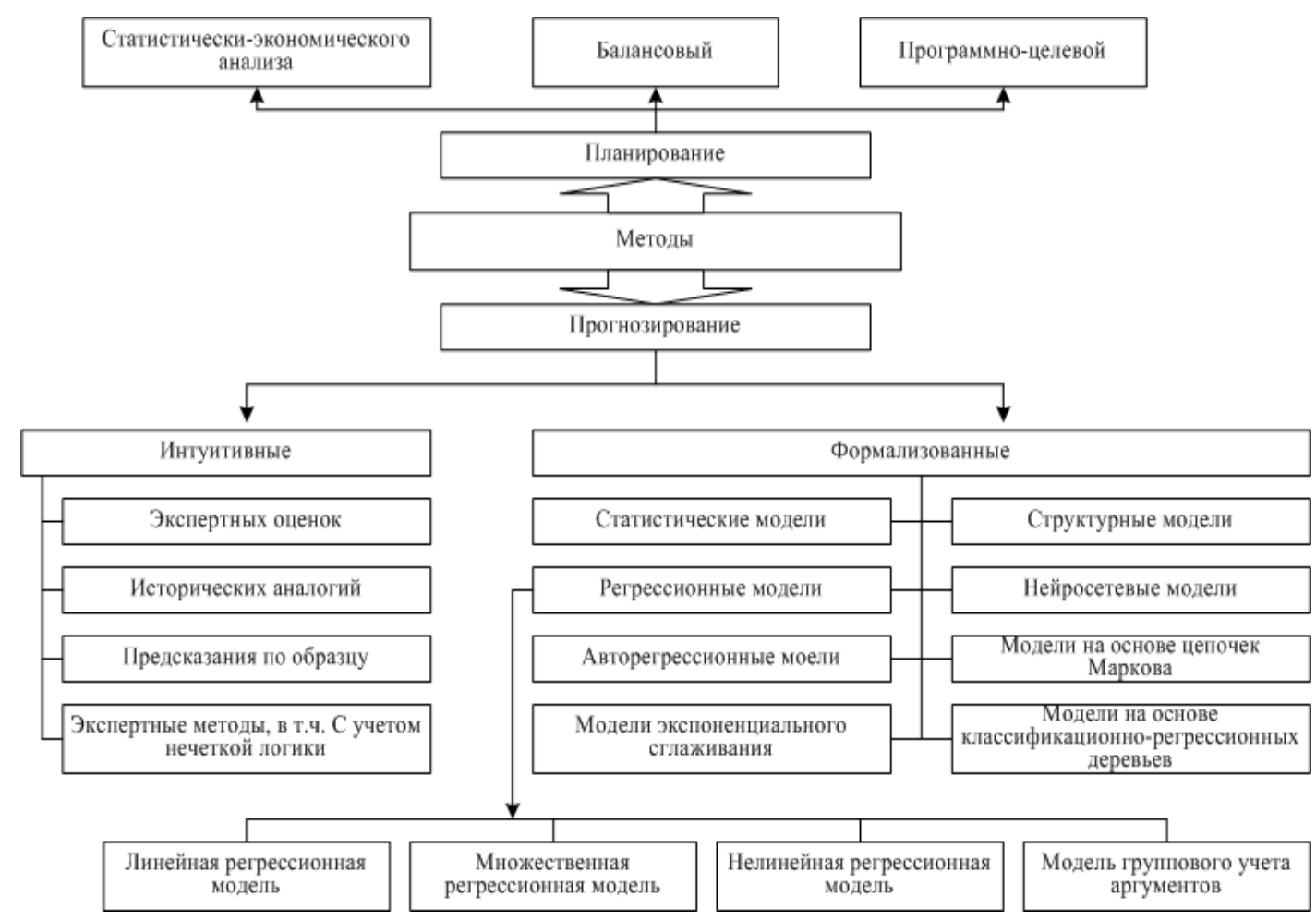

Рис. 3. Систематизация научных методов прогнозирования

и пианирования социамьно-экономических процессов

Примечание: составлено автором на основе (Gonta, 2015, Grachova, 2013, Danilov, Zin, 2007)

К преимуществам таких моделей следует отнести простоту, гибкость, а также еАиный поАхоА к их анализу и проектированию (Draper, 1981). В частности, использование минейной регрессии позволяет получить результат прогнозирования значительно быстрее, чем при применении других модемей. Кроме этого, преимуществом данного метода выступает прозрачность процесса моделирования (Mazengia, 2008), что выражается в доступности Аця анализа всех промежуточных вычислений. СреАи недостатков минейных регрессионных моделей необходимо отметить низкую адаптивность и отсутствие возможности моделирования нелинейных процессов (Catalão, 2007).

При исслеАовании факторов, вмияющих на структуру электроэнергетики государства, можно использовать несколько подходов, среАи них наиболее распространенными выступают (Kalyuzhnaja, 2015, Korikov, 2005, Surmin, 2003):

- дескриптивный - метоА, позвомяющий описательно определить факторы, которые оказывают наибольшее влияние на структуру всей электроэнергетики. В рамках этого подхода отрасль рассматривается как система;

- конструктивный - направление, руководствуясь которым можно построить модель факторов влияния на структуру электроэнергетики страны как системы. То есть, структура Аанной отрасли будет определена как система на основе функционально-целевого подхода. Аанный объект исследования необходимо рассматривать как комплексную модель, состоящую из соответствующего перечня факторов развития эмектроэнергетики. Кроме того, на конструктивный подхоА Аает возможность определения и обоснования показателей, которые больше всего влияют на совершенствование работы отрасли.

Применение данных методик позволяет провести типологизацию элементов системы путем включения их в соответствующий тип с целью Аальнейшего исследования.

В основу конструктивного метода положена регрессионная (многофакторная) модель, на основе которой можно Аать компиексную оценку факторам вмияния на структуру электроэнергетики государства и определить наиболее значимые из них.

Регрессионный анализ проводится на основе построенного уравнения регрессии и позволяет оценить вклаА кажАой независимой переменной в вариацию исследуемой (прогнозируемой) зависимой переменной величины. Основная задача регрессионного анализа заключается в определении влияния факторов на результативный показатель. Аця этого необхоимо подобрать и обосновать уравнения связи, соответствующей характеру анахитической стохастической зависимости межАу исследуемыми признаками. Уравнение регрессии показывает, как 
в среАнем изменяется результативный признак $\left(Y_{x}\right)$ поА влиянием изменения факторных признаков $\left(X_{i}\right)$.

Цель государственного регулирования электроэнергетики, как составмяющей макроэкономической политики в контексте структурно-отраслевой стратегии развития национамьной экономики, опреАемяется с помощью показателей, которые выступают индикаторами общего экономического состояния хозяйственных процессов в эмектроэнергетической сфере страны. ОАним из основных показателей макроуровня, который опредемяет структуру электроэнергетики и представляет результирующую оценки экономической Аеятельности субъектов электроэнергетического рынка, выступает количество произвеАенной электроэнергии Аля промышменных и бытовых цемей.

\section{5. ВЫводы}

На основании проведенного обзора научной митературы доказано, что задача построения прогнозов приобретает высокую актуальность во многих преАметных областях и выступает неотъемлемой компонентой повсеАневной работы современных социально-экономических систем.

Решение текущих и стратегических вопросов госуАарственного регулирования и поиск информационно-аналитического обеспечения выполнения возможенных функций на аппарат регулирования требует применения прогностических метоАОв и поАХоАОв Аля сохранения Аинамического развития рынка эмектрической энергии и соблюдения сбалансированности интересов всех его субъектов в условиях реформирования.

Аальнейшие исследования будут посвящены изучению особенностей применения корремяционно-регрессионного подхода Аля выявления кмючевых факторов, определяющих результативный показатель - объемы производства электрической энергии в стране, Y, а также анализу их взаимного вмияния на основе результатов моделирования.

\section{References:}

Otchet o rezultatah deyatelnosti Natsionalnoy komissii, osuschestvlyayuschey gosudarstvennoe regulirovanie $\mathrm{v}$ sferah energetiki i kommunalnyih uslug, v 2016 godu [The Report on the activity results of the of the National Commission implementing state regulation in the energy and utilities sectors in 2016], utverzhden Postanovleniem NKREKU ot 30.03.2017 № 460. Retriever from: http://www.nerc.gov.ua/data/filearch/Catalog3/Richnyi zvit NKREKP 2016.pdf. - 292 p.

Ukrayina poky ne zbirayetsya vidnovlyuvaty eksport elektroenergiyi do Bilorusi ta Moldovy (2015) [Ukraine is not going to resume electricity exports to Belarus and Moldova]. UNIAN. Ekonomika. Energetika. Retriever from: http://economics.unian.ua/energetics/1031690-ukrajina-poki-ne-zbiraetsya-vidnovlyuvati-eksportelektroenergiji-do-bilorusi-ta-moldovi.html.

Gonta, I. (2015). Neozhidannyj energoudar [Unexpected energy attack]. Mirror of the week. Retriever from: http://gazeta.zn.ua/energy_market/neozhidannyy-energoudar-otkaz-ot-zelenoy-alternativy-netenergeticheskoy-nezavisimosti-_.html

Grachova, E. I. (2013). Prognozirovaniye poter elektroenergiyi v setyah nizkogo napryazheniya na osnove regressionnogo analiza [Forecasting of electricity losses in low voltage networks based on regression analysis]. The Bulletin of the South Ural State University. Series: Energy, Vol. 2, T. 13, pp. 19-24.

Danilov, I. A. Operezhayushcheye razvitiye elektroenergetiki neobhodimoye usloviye razvitiya ekonomicheskoy modeli [Advanced development of the electric power industry is a prerequisite for the development of the economic model]. Retriever from: http://www.eeseaec.org/vestnik-energetiki-eaec/stati-otzyvy-kommentarii-prodolzenie Zin, E. A. (2007). Regionalnaya ekonomika [Regional economy]: uchebnik - Kijev, ProfesIonal, 528 p.

Kalyuzhnaja, N. G. (2015). Opredeleniye ponyatiy «sistema» i «sistema upravleniya» na osnove deskriptivnogo i konstruktivnogo podhodov [Definition of the concepts "system" and "management system" on the basis of descriptive and constructive approaches]. Biznes Inform, issue 2, pp. 15-20.

Korikov, A. M., Safyanova, O. M.(2005). Osnovy sistemnogo analiza i teorii sistem [Fundamentals of system analysis and system theory] - Tomsk, TGU, 320 p.

Leonenkov, A. V. (2005). Nechetkoye modelirovaniye v srede MATLAB fuzzyTECH [Fuzzy modeling in MATLAB environment fuzzyTECH] - Spb, BHV-Peterburg, $736 \mathrm{p}$.

Muratova, L. I., Bakov N. H., Horuzhiy V. I., Kanukov V. M. (2009). Normativnye sistemy v prognozirovanii razvitiya predprinimatelskogo sektora ekonomiki (Part 1) [Normative systems in forecasting the development of the business sector of the economy]. Upravlenie ekonomicheskimi sistemami, issue 20. Retriever from: http://www.uecs.ru/predprinematelstvo/item/142--1

Surmin, Yu. P. (2003). Teoriya sistem i sistemnyi analiz [The Theory of systems and systems analysis]: ucheb. posob. - Kiyev, MAUP, 368 p.

Tihonov, E. E. (2006). Prognozirovaniye v usloviyah rynka: uchebnoe posobie [Market Forecasting] Nevinnomyissk, $221 \mathrm{p}$.

Catalão, J. P. S., Mariano, S.J.P.S., Mendes, V.M.F., Ferreira, L.A.F.M. (2005). An artificial neural network approach for day-ahead electricity prices forecasting, Trabalho apresentado em NN'05, In Proceedings of the 6th WSEAS International Conference on Neural Networks, Lisbon, Portugal, Issue 4., Vol. 4, pp. 451-454. 
Catalão, J. P. S., Mariano, S.J.P.S., Mendes, V.M.F., Ferreira, L.A.F.M. (2007). An artificial neural network approach for short-term electricity prices forecasting, Engineering Intelligent Systems, issue 15, Vol. 1, pp. 15-23.

Cuaresma, J. C., Hlouskova, J., Kossmeier, S., Obersteiner, M. (2004). Forecasting electricity spotprices using linear univariate time-series models. Applied Energy, Issue 77(1), pp. 87-106.

Draper, N., Smith, H. (1981). Applied regression analysis - New York, Wiley, In press, 693 p.

Escribano, A, Pe na, J. I. and Villaplana, P. (2002). Modelling electricity prices: International evidence. Working Paper 02-27, Universidad Carlos III de Madrid.

Fezzi, C. (2007). Econometric models for the analysis of electricity markets. Phdthesis in statistical methodology for scientific research, XIX cycle, Department ofStatistics, University of Bologna, $166 \mathrm{p}$.

Jingfei Yang, M. Sc. (2006). Power System Short-term Load Forecasting: Thesis for Ph.d degree, Germany, Darmstadt, Elektrotechnik und Informationstechnik der Technischen Universität, 139 p. Retriever from: http://tuprints.ulb.tu-darmstadt.de/epda/000662/Yangjingfei.pdf

Kristiansen, T. (2012). Forecasting NordPool day ahead prices with an autoregressive model. Energy Policy, issue 49, pp. 328332.

Mazengia, D. H. (2008). Forecasting spot electricity market prices using time series models: Thesis for the degree of Master of Science in Electric Power Engineering, IEEE International conference on Sustainable Energy Technologies, pp. 1256-1261.

Murthy, G. G. P., Sedidi, V., Panda, A. K., Rath, B. N. (2014). Forecasting Electricity Prices in Deregulated Wholesale Spot Electricity Market: A Review. International Journal of Energy Economics and Policy, Vol. 4, issue 1, pp. 32-42. Nan, Fany (2009). Forecasting next-day electricity prices: from different models to combination, $130 \mathrm{p}$.

Panagiotelis, A. and Smith, M. (2008). Bayesian density forecasting of intraday electricity prices using multivariate skew $t$ distributions. International Journal of Forecasting, issue 24, pp. 710727.

Szkuta, B. R., Sanabria, L. A. and Dillon, T. S. (1999). Electricity Price Short-Term Forecasting using Artificial Neural Networks. IEEE Trans. Power Syst., issue 14(3), pp. 851857.

\section{Анастасия КОЛЕСНИЧЕНКО}

\section{ТЕОРЕТИЧЕСКИЕ АСПЕКТЫ ПРИМЕНЕНИЯ ПРОГНОСТИЧЕСКОГО ИНСТРУМЕНТАРИЯ В ОБЛАСТИ ГОСУДАРСТВЕННОГО РЕГУЛИРОВАНИЯ ВЗАИМООТНОШЕНИЙ СУБЪЕКТОВ РЫНКА ЭЛЕКТРОЭНЕРГИИ}

Аннотация. Современные тенденции развития электроэнергетики в условиях нарастания концентрации капитала, информатизации экономики и повышения динамики движения значительных объемов денежных потоковтребуетперманентногоанализатекущейситуацииинеобходимойкорректировкии/илимодификации параметров регулирования энергорынков. Задача построения прогнозов приобретает высокую актуальность во многих предметныхобластях и выступает неотъемлемой компонентой повседневной работы современных социально-экономических систем, одним из важнейших институтов которых выступают взаимоотношения субъектов рынка электрической энергии. Целью данной статьи выступает исследование теоретической базы касательно адаптивного применения методов прогнозирования в области функционирования энергорынков. Во избежание доминирования коммерческих интересов определенных групп участников энергорынка, появления ценовых перекосов на рынке, которые оказывают деструктивное воздействие на получение потенциальных выгод от внедрения конкурентных механизмов, ключом к поиску максимально эффективного и экономичного пути решения данных проблем выступает применение системного регулирования возникающих отклонений при достижении целевых ориентиров, особенно в условиях реформирования. Формирование информационно-аналитического обеспечения выполнения возложенных функций на аппарат регулирования требует применения прогностических методов и подходов для сохранения динамического развития рынка электрической энергии и соблюдения сбалансированности интересов всех его субъектов в условиях реформирования. В статье использован рядметодов: классификации и систематизации при анализе научных методов прогнозирования и планирования социально-экономических процессов; логического обобщения при обзоре существующих методов моделирования и оценке их эффективности в области энергорынков; обосновано применение конструктивного подхода при исследовании факторов, влияющих на результирующий показатель моделирования. Полученные теоретические и научно-прикладные результаты исследования позволяют сформировать необходимую теоретическую и методическую базу для усовершенствования инструментальной основы моделирования взаимоотношений субъектов рынка электрической энергии, которую можно использовать при прогнозировании в области регулирования явлений и процессов энергорынков. 